\title{
Diseño de sistema de calentamiento para la simulación del flujo de plasma
}

Design of a heating system for plasma flux simulation

\author{
Juan Carlos Fallas-Chinchilla' \\ Manuel Fallas-Agüero ${ }^{2}$ \\ Juan Ignacio Del Valle-Gamboa ${ }^{3}$ \\ Luis Diego Fonseca-Flores ${ }^{4}$
}

Fecha de recepción: 10 de octubre del 2012

Fecha de aprobación: 30 de enero del 2013

Fallas-Chinchilla J; Fallas-Aguero M; Del Valle-Gamboa J; Fonseca-Flores L. Diseño

de sistema de calentamiento para la simulación del flujo de plasma Tecnología en Marcha. Vol. 26, № 3. Pág 3-12

Ingeniero Mecánico. Egresado del programa de Maestría en Ciencia e Ingeniería de Materiales, Universidad de Nevada, Reno. Costa Rica. Correo electrónico: juancarlosfallas@yahoo.com

2 Profesor retirado, Escuela de Ingeniería Mecánica, Universidad de Costa Rica.Costa Rica. Correo electrónico: manuel.fallas@ucr.ac.cr

3 Ingeniero de análisis térmico, Ad Astra Rocket Company, Costa Rica. Correo electrónico: juan@adastrarocket.com

4 Ingeniero de análisis térmico, Ad Astra Rocket Company, Costa Rica. Correo electrónico: diegox@adastrarocket.com 


\section{Palabras clave:}

VASIMR; motor de plasma; Ad Astra Rocket Company; tubo de gases

\section{Resumen}

El motor de plasma VASIMR® y sus futuras aplicaciones está causando una revolución en el transporte espacial. Ad Astra Rocket Company, la empresa encargada de su creación, requiere instrumentos que permitan evaluar los componentes del motor, para recopilar información y tener certeza de un funcionamiento seguro. Este artículo describe el diseño y construcción de un sistema de calentamiento, el cual simula el flujo de gas que será ionizado a alta temperatura para convertirse en plasma dentro de un tubo cerámico. Esta parte interna del VASIMRß cumple esta tarea para la respectiva propulsión. Se establecieron restricciones en el diseño para acercar más el sistema de simulación a las condiciones de trabajo en el espacio. El ubicar el sistema dentro de una cámara de vacío y modelar adecuadamente el calentamiento en el conducto cilíndrico producto del flujo de plasma, muestran dicho acercamiento. La herramienta se instaló en la sede de la empresa, en Costa Rica, donde se utiliza para establecer el comportamiento del tubo a estas altas temperaturas y obtener datos experimentales. Posteriormente se podrá detallar un modelado asistido por computadora.

\section{Key words:}

VASIMR; plasma engine; Ad Astra Rocket Company; gases tube

\begin{abstract}
VASIMRß plasma rocket and its possible applications are promoting innovations in the space propulsion area. Ad Astra Rocket Company (VASIMRß designer) constantly needs instruments to evaluate the plasma engine and its components, in order to acquire critical information to warrant a safe operation. This study describes the design and construction of a heating system, to simulate a plasma flux within a ceramic tube. This part of the engine plays a critical role, transporting a gas to ionize at high temperatures, transforming it in plasma for propulsion purposes. In the simulation system, some restrictions were established to match its labor with the tube and its operation conditions in the space. Making the simulation inside a vacuum chamber to remove the air, and adapting the heaters in a specific position to relate them with the real plasma flux and its behavior, are examples of the simulation requirements. The simulation system was installed in Ad Astra Rocket Company, Costa Rica. It is used to acquire data and study the behavior of the tube under the previously mentioned conditions, and detail a computer-assisted model.
\end{abstract}

\section{Introducción}

Frank-Kamenetskii (1972) explica la naturaleza del plasma como gas ionizado, o gas con conductividad eléctrica, que puede ser contenido en "recipientes" magnéticos, siendo confinado a las líneas de campo magnético. Bajo el mismo principio, Díaz (2005) indica que se puede crear una configuración de las líneas de campo magnético que permita acelerar el plasma a muy altas velocidades.

El motor de plasma VASIMRß se basa en el fenómeno de aceleración previamente indicado. Fallas (2007) describe el funcionamiento del motor VASIMR y sus diferentes etapas en detalle. Su funcionamiento se inicia cuando el gas por ionizar ingresa a un sistema de inyección que regula su flujo, pasando luego a un tubo cerámico que confina el gas (llamado tubo de gases), para así llegar a la primera antena de radiofrecuencias (antena Hélicon), donde un haz de radiofrecuencias traspasa el tubo de gases y hace que las moléculas del gas vibren y se calienten para ionizarse y formar el plasma.

Dentro de la investigación y desarrollo en la empresa Ad Astra Rocket, con sede en Costa Rica, se estudia el desempeño de nuevos materiales, para mejorar la durabilidad de los componentes asociados al motor de plasma. Uno de los objetivos es probar materiales diferentes al cuarzo $\left(\mathrm{SiO}_{2}\right)$, usado actualmente en el tubo de gases. La alúmina $\left(\mathrm{Al}_{2} \mathrm{O}_{3}\right)$, se considera una alternativa importante para tomar en cuenta. Considerando que en Costa Rica las fuentes estables de plasma para 
propulsión se encuentran en una etapa temprana, se debe implementar una herramienta para evaluar el comportamiento del tubo de gases.

Se construyó un sistema de simulación para mostrar los efectos que la temperatura del plasma tendrá en esta pieza clave. En síntesis, con este sistema de simulación (también llamado sistema de calentamiento) se podrá practicar una toma de datos experimental, que cuantifique el comportamiento del material y su reacción a altas temperaturas. Posteriormente, los datos se podrán utilizar en un programa computacional basado en elementos finitos, teniéndose entonces una base teórica y práctica que extrapole el conocimiento generado y aproxime la operación del tubo a las temperaturas mucho más altas que el sistema construido pueda ofrecer.

Las restricciones en este proyecto buscan que el sistema de calentamiento pueda funcionar dentro de una cámara de vacío y acercarse al máximo al escenario real de operación del motor ya mencionado. Entre otros requerimientos en los que debe operar el artefacto se mencionan las siguientes:

I. Debe modelar adecuadamente el calentamiento que ocurre dentro del conducto cilíndrico producto de la generación de plasma.

2. La cantidad de energía debe ser de 200 Watts para un uso nominal, además, debe poseer funciones de regulación para su aumento o disminución.

3. El método de transferencia de calor debe darse por la conducción y/o la radiación, dada la operación al vacío.

4. La localización de la fuente de calor debe tener la posibilidad de calentar diferentes zonas del tubo cerámico, ya sea únicamente el centro, únicamente sus extremos, o la extensión completa del tubo. Con esto se podrá tomar en cuenta la antena Hélicon y su ubicación en el medio del tubo de gases.

5. El dispositivo se debe adaptar a los requerimientos geométricos del conducto, el cual posee como diámetros interno y externo 70 $\mathrm{mm}$ y $80 \mathrm{~mm}$ respectivamente, así como un metro de longitud.

6. Las conexiones eléctricas y de toma de datos se deben ajustar a la cámara de vacío. Se debe realizar una selección de los componentes y las piezas adecuadas para adaptarse a ella.
7. Se debe considerar una prevista en el calentador para la toma de datos por medio de un ordenador.

Según Chang Díaz (200I), las proyecciones indican que el tubo de gases en estudio tendrá una temperatura de uso continuo de aproximadamente $600{ }^{\circ} \mathrm{C}$.

\section{Diseño del sistema de calentamiento}

Los calentadores eléctricos son ideales para cumplir con las restricciones enumeradas. Estos constan fundamentalmente de resistencias eléctricas, fabricadas con Nicromio. Dentro de la gama que ofrece el mercado costarricense, se apreciaron como la mejor opción en este diseño. Su alta tolerancia a la temperatura, su bajo costo y disponibilidad en el país, hacen que este tipo de elemento calefactor sea muy versátil para este caso. La posibilidad de manufactura de las resistencias en forma helicoidal a la medida del diámetro interno del tubo favoreció su colocación, pues las resistencias se configuraron para que las espiras se encuentren en contacto con el tubo de alúmina y le cedan una mayor cantidad de calor por conducción.

El Nicromio utilizado para esta clase de aditamentos eléctricos es el Nicromio 60. El fabricante de alambre MOR (2007) describe en amplitud sus características y composición. Para el calentamiento independiente de las zonas laterales y central del tubo de gases fue necesario colocar tres resistencias, donde un sistema de control fue el encargado de proporcionar la dosificación de potencia adecuada en cada tramo del tubo que se requiera en la parte experimental. Debido a que se busca maximizar las zonas de calentamiento dentro del tubo de gases, cada una de las tres resistencias deberá tener una longitud de $30 \mathrm{~cm}$ y los dos aislantes cerámicos una de $5 \mathrm{~cm}$ para evitar el contacto entre ellas.

El sistema de control añade también funciones útiles a la hora de experimentar con este conjunto calentador. Se citan las siguientes: I) botones de encendido y de apagado del sistema, 2) luz indicadora de encendido, 3) potenciómetros que regulan la entrega independiente de potencia para las resistencias laterales y central, 4) disipadores de calor para evitar el sobrecalentamiento de los componentes electrónicos del sistema, 5) borneras para el ordenamiento del cableado de alimentación 
de las resistencias, 6) control remoto. La última de estas características obedece a que, durante las pruebas experimentales, las resistencias estarán dentro de la cámara de vacío, a unos metros de la mesa de trabajo. El diagrama eléctrico del sistema de control se basa en los sistemas de regulación de potencia tipo Dimmer (utilizado en iluminación casera para regular la intensidad de las luces de una habitación). Fallas (2007) precisa detalles acerca de esta clase de dispositivos.

El sistema de control se divide en etapa de control y etapa de potencia. La etapa de control es la encargada de enviar las señales de encendido, apagado y las de

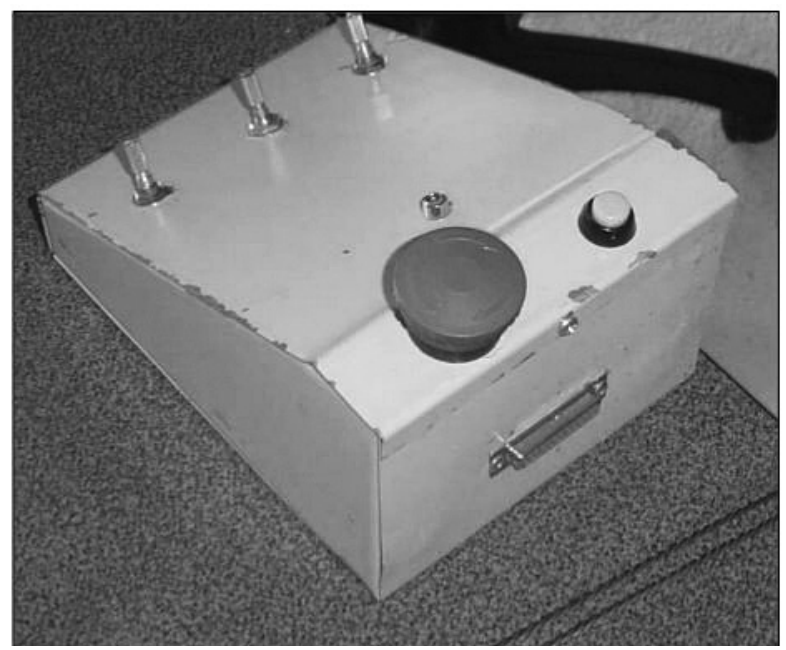

Figura I. Etapa de control. Fuente: Fallas (2007).

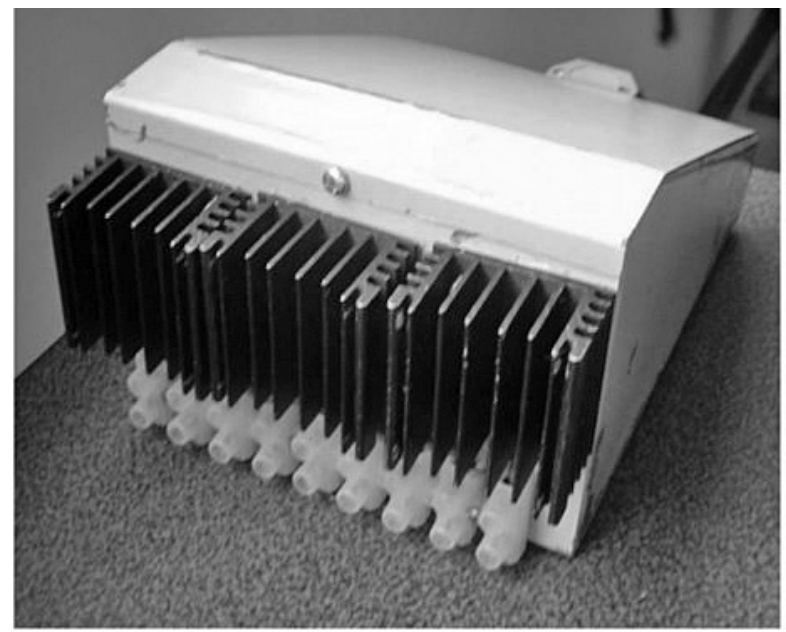

Figura 2. Etapa de potencia. Fuente: Fallas (2007). los potenciómetros a cada una de las resistencias (encargados de la regulación de la potencia). Por la naturaleza de estas señales, se pueden llevar a un Relé por medio de cable o alambre delgado. En este caso se utilizó el cable UTP para conformar el llamado control remoto del sistema. Cuando el cable llega a la etapa de potencia y se conecta con el Relé, este componente traduce estas débiles señales de control en conexiones que manejen corrientes y voltajes más altos, para así activar las resistencias y regular la entrega de potencia.

Las figuras I y 2 muestran ambas etapas del sistema de control dentro de una caja metalica para su debida protección.

\section{Diseño de las resistencias}

El fabricante de resistencias Hyndman (200 I) explica las propiedades de los calibres típicos del alambre de Nicromio. Se escogió utilizar el calibre 15, el cual posee una resistividad de 0,680 Ohms por metro. Las siguientes relaciones matemáticas serán vitales para el diseño de las resistencias:

$$
\begin{aligned}
& P=V I \quad(I) \\
& P=I^{2} R \quad(2)
\end{aligned}
$$

Donde "V" corresponde a voltaje (voltios), "I" a corriente (amperios), "R" a resistencia (ohmios) y "P" a potencia (watts).

Para calcular la potencia máxima de cada una de las resistencias, se tomaron los valores de trabajo del sistema de control como punto de referencia. Este posee como parte crítica los TRIACS, elemento que tiene un límite superior de operación de 240 voltios y 10 amperios para este caso específico. El sistema de control utilizará 120 voltios de la red eléctrica costarricense, y se propuso como límite de funcionamiento 8 amperios para evitar problemas de calentamiento en los TRIACS. Al usar la ecuación ( I) con el voltaje y corriente ya definidos, se nota que la potencia máxima que el sistema de control puede manejar es de 960 Watts.

Por lo anterior, se fabricaron las resistencias para 650 Watts de potencia, cubriendo tanto la necesidad de 200 Watts nominales que la empresa solicita como potencias mayores en caso de que se deseen simular temperaturas más altas. Al tener el alambre de Nicromio elegido un valor de 0,680 Ohms por 
metro, y utilizando la ecuación (2) con los valores de 8 amperios para la corriente, y el de 650 Watts, cada resistencia posee aproximadamente 10,15 Ohmios, necesitando |4,92 metros de alambre de Nicromio calibre 15 en cada resistencia.

\section{Fabricación de las resistencias}

Las resistencias fueron manufacturadas en ElectroMAZ S.A. (San José, Costa Rica). La Figura 3 ejemplifica las resistencias y sus partes. Las resistencias laterales poseen dos alambres principales para la alimentación eléctrica, uno al inicio y otro al final de la resistencia. Los dos alambres deben salir por la abertura del tubo más cercana, el alambre del final debe retroceder por dentro del cuerpo de la resistencia.

La resistencia central tiene la particularidad de que sus dos alambres de alimentación deben salir del tubo pasando por el interior de las resistencias laterales.

Se hizo necesario incorporar aislantes, ya que el contacto entre los cables de alimentación de una misma resistencia o entre las espiras de resistencias diferentes provocaría un cortocircuito. El aislante cerámico individual (conocido como aislante tipo perla), consiste en una serie de segmentos cerámicos muy pequeños que se colocan en los alambres con el fin de que no tengan un contacto directo al estar energizados y se genere un cortocircuito. El aislante cerámico individual también tiene la función de evitar que se funda este alambre de alimentación por el calor del funcionamiento, y también se puede apreciar en la Figura 3. Fuera del tubo, las resistencias toman una forma arbitraria, sin embargo, están diseñadas para que cada una tenga aproximadamente una extensión de $30 \mathrm{~cm}$ y una separación de $5 \mathrm{~cm}$ entre las laterales y la central dentro del tubo. El fabricante tomó en cuenta la expansión que sufren los metales a la hora de que su temperatura aumenta, para que las espiras estén en contacto con la pared interna del tubo. Se decidió realizar el proceso de "ingeniería hacia atrás", el cual consiste en el análisis de un producto fabricado con el fin de verificar que el diseño sea el correcto.

La cantidad de alambre que posee la resistencia central equivale a 1 109,8 I Watts y las laterales a 84I,92 Watts.

Se favorece al sistema de calentamiento, pues se tiene aún más potencia que los 650 Watts planteados. El sistema de control puede manejar la potencia de las resistencias laterales, no así en la resistencia central pues excede el límite de 960 Watts, quedando un remanente sin uso.

\section{Instalación del sistema de calentamiento}

Primeramente, se tomó en cuenta que, al estar comprimida una resistencia, hay probabilidades de que algunas espiras entren en contacto. Este factor no

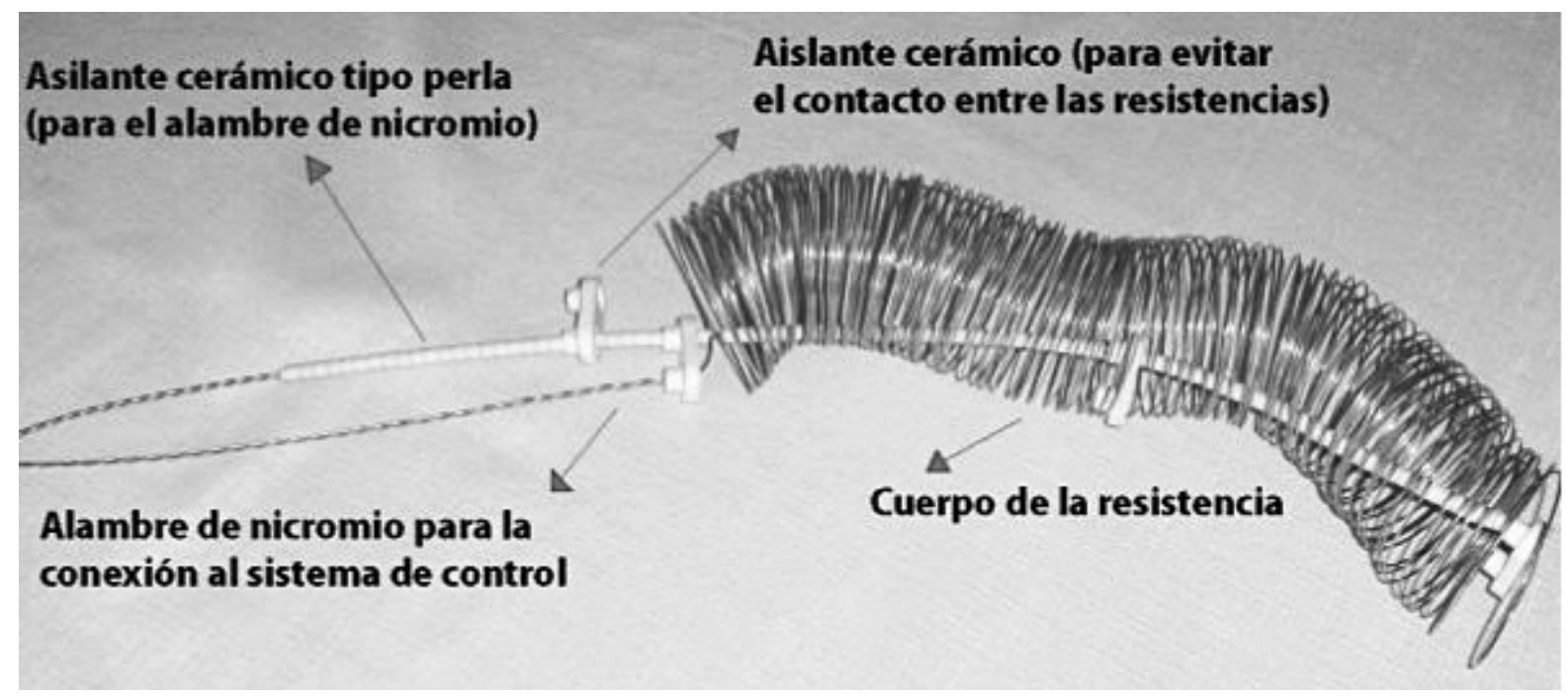

Figura 3. Detalle de las resistencias y sus partes. Fuente: Fallas (2007). 
es tan crítico como si se tratara de dos espiras de diferentes resistencias, sin embargo, se genera un cortocircuito, en el cual las espiras que se encuentren en unión tendrán valores de resistencia casi nulos, siendo afectada la transferencia de calor. El sistema se colocó dentro de la cámara de vacío de la empresa Ad Astra Rocket en Costa Rica. El tubo fue fijado a la cámara por medio de los soportes fabricados para el caso. Estos fueron sujetados a los postes horizontales inferiores de la cámara por medio de prensas metálicas. En la Figura 4 se observa la fijación de los soportes y del tubo de alúmina a ellos.

El paso siguiente fue la puesta de los adaptadores para vacío, los cuales serán los encargados de que los cables de alimentación eléctrica y de toma de datos puedan entrar a la cámara sin provocar problemas de estanqueidad. Existen adaptadores de este tipo tanto para salida de datos (temperatura, en este caso), como para el ingreso de potencia para la alimentación del sistema de resistencias eléctricas.

Debido a que el sistema de control está adecuado para trabajar con 120 voltios de corriente alterna y con un máximo de 8 amperios, se procede a seleccionar uno acorde con esas necesidades, así como también un número de pines razonable, ya que estos serán los encargados de realizar las conexiones.

Cada resistencia posee dos conexiones, por lo que el número mínimo de pines es de 6. El modelo EFT0082033, ofrecido por el fabricante de aditamentos para vacío Lesker (2006), tiene 8 pines y

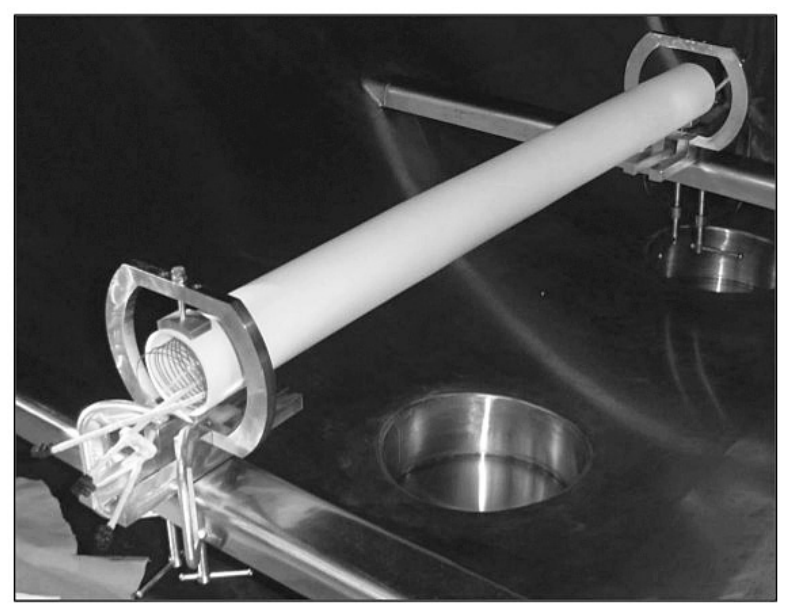

Figura 4. Soportes del tubo de alúmina y tubo de alúmina instalado. Fuente: Fallas (2007). una capacidad de hasta 500 voltios y 10 amperios, por lo que la selección es adecuada. Los adaptadores para vacío para la toma de datos están provistos de termopares tipo $\mathrm{K}$, y tienen un rango de mediciones de temperatura desde $0{ }^{\circ} \mathrm{C}$ hasta $1370{ }^{\circ} \mathrm{C}$ aproximadamente. Como se decidió medir la temperatura del tubo de alúmina en 10 puntos diferentes, se realizó la selección de los adaptadores correspondientes. El modelo TFT5KY00003 posee cinco pares de medición, por lo que se necesitan dos para satisfacer las necesidades (Lesker, 2006). El mismo fabricante ofrece más detalles de estos instrumentos.

En la Figura $5 a$ se observa la forma en que este cableado es llevado a un dispositivo de captura de datos, el cual los envía a la computadora principal para un adecuado registro. Se observa en esa misma imagen la etapa de potencia del sistema de control, ya que es donde los cables de alimentación del adaptador de vacío de potencia son llevados para que se realice su regulación. La Figura 5b muestra el acomodo externo de los adaptadores para vacío y su respectivo cableado. Se continuó con la parte del tubo, en lo que respecta al cableado de alimentación y a la fijación de los termopares de los adaptadores para vacío para la toma de datos. La Figura 6 (a) muestra la configuración hecha para el cableado de alimentación y toma de datos. Se instaló una bornera de un material cerámico para que resista una mayor temperatura y se realizó la fijación de los termopares al tubo. Los cables de estos fueron guiados y ordenados por medio de una placa adaptada a los soportes del tubo, la cual está provista de una serie de conductos cilíndricos que llevan los termopares directamente hacia el tubo. Se fijó cada termopar al tubo por medio de una pasta cerámica especial, diseñada para soportar tanto el vacío como el calor que se produzca. Cuando se revisó meticulosamente la instalación de todo este complejo sistema, se procedió a cerrar la cámara de vacío para iniciar las pruebas de simulación de flujo de plasma, como muestra la Figura 6b.

\section{Desarrollo del experimento}

Para observarel sistema de calentamiento cumpliendo su labor, se practicaron dos corridas experimentales. La toma de datos se realizó con ayuda del programa $\mathrm{VI}$-Logger $\AA$ de National Instruments $\AA$, el cual brinda no solo los datos de temperatura de cada 

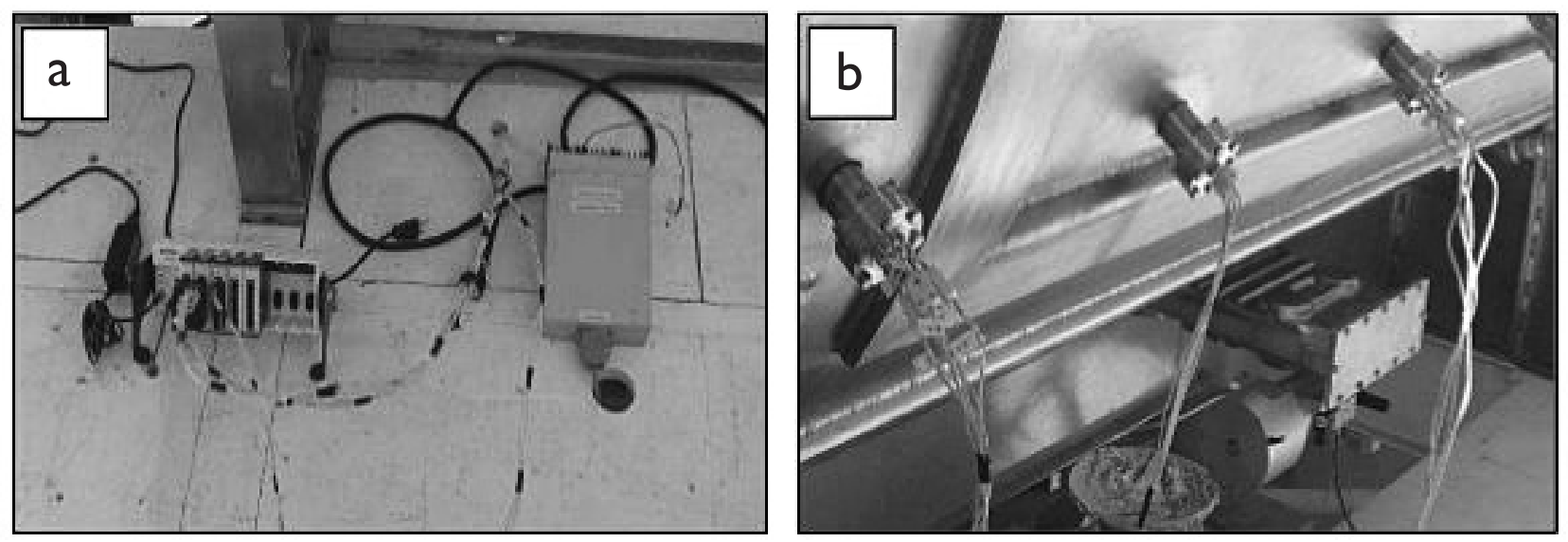

Figura 5. a) Cableado de toma de datos; b) adaptadores para vacío instalados (b). Fuente: Fallas (2007).
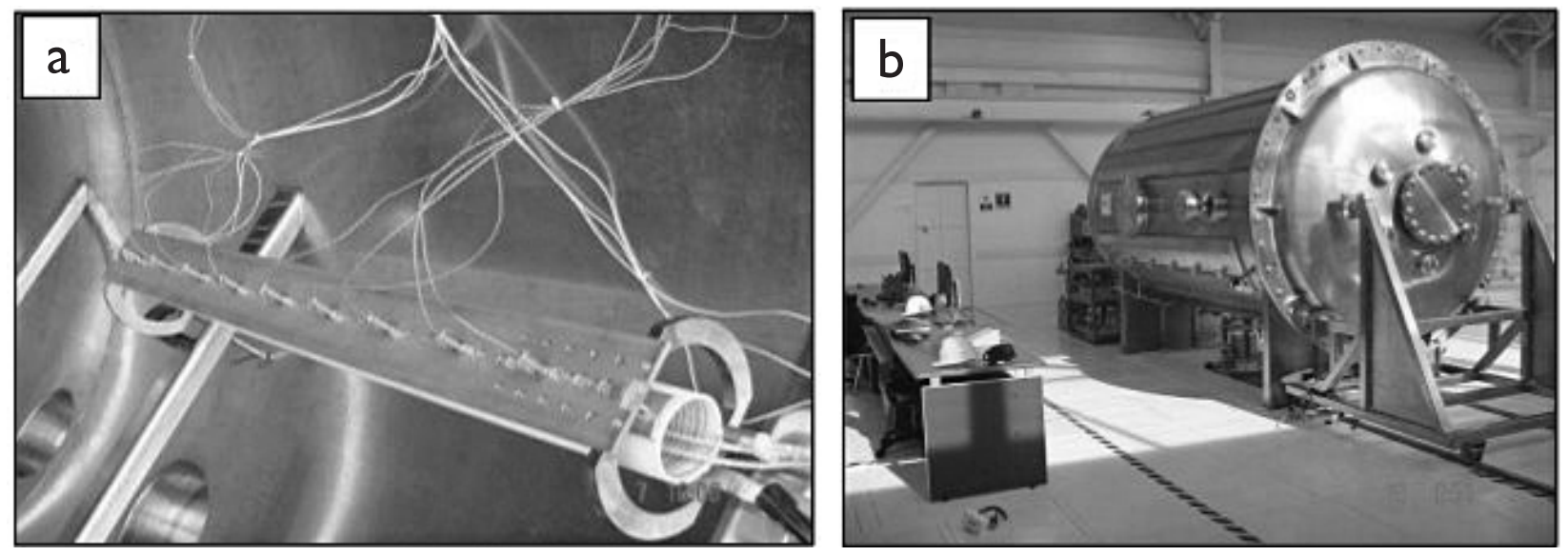

Figura 6. a) Cableado de alimentación de las resistencias; b) Cámara de vacío y mesa de trabajo. Fuente: Fallas (2007).

termopar sino que también muestra gráficamente la progresión que se tiene durante la puesta en marcha de las resistencias. Mientras los ingenieros de Ad Astra Rocket recopilaban los datos, tomaban el tiempo y controlaban el sistema desde la mesa de trabajo, los valores de voltaje y de corriente eran medidos directamente en el sistema de control con un voltímetro y un amperímetro. En la corrida inicial, se establecieron por cada resistencia cuatro minutos de trabajo y dos amperios aproximadamente, bajo la condición de vacío. Los datos que se recopilaron se reflejan en el Cuadro I. La Figura 7 muestra el resultado que se tuvo en termopares seleccionados durante este primer ensayo. La segunda corrida se realizó con iguales parámetros de tiempo y amperaje, pero con presión atmosférica para hacer una comparación entre estas dos condiciones de uso del tubo. Los datos recopilados se muestran en el Cuadro 2 y la Figura 8.

Para analizar los resultados, se debe indicar primeramente que la escala tanto de tiempo como de temperatura ha sido suprimida, puesto que los datos recopilados son propiedad de Ad Astra Rocket Company. Sin embargo, cabe la posibilidad de mencionar que las temperaturas superiores rondaron los $100^{\circ} \mathrm{C}$. La presencia de aire alrededor del tubo cerámico contribuye a que la temperatura de los termopares aumente en menor tiempo que la registrada para el caso al vacío. El aire facilita el transporte de calor por convección, lo que crea una mayor velocidad en la transferencia de calor y este se traslada por el resto del tubo con mayor facilidad. Al finalizar el tiempo de prueba, se nota también como las temperaturas convergen hacia la ambiental, para recuperar su equilibrio una vez 
Cuadro I. Primera corrida del experimento.

\begin{tabular}{|c|c|c|c|c|}
\hline \multicolumn{5}{|c|}{ Prueba 2 (Vacío) } \\
\hline Orden & $\begin{array}{l}\text { Duración } \\
\text { (minutos) }\end{array}$ & $\begin{array}{l}\text { Corriente } \\
\text { (amperios) }\end{array}$ & $\begin{array}{c}\text { Voltaje } \\
\text { (voltios) }\end{array}$ & $\begin{array}{r}\text { Potencia } \\
\text { (Watts) }\end{array}$ \\
\hline Resistencia central & 4 & 1,9 & 51,5 & 97,85 \\
\hline Resistencia lateral & 4 & 1,9 & 108,6 & 206,34 \\
\hline Resistencia lateral & 4 & 2 & 54,2 & 108,4 \\
\hline
\end{tabular}

Cuadro 2. Segunda corrida del experimento.

\begin{tabular}{|r|c|c|c|c|}
\hline \multicolumn{5}{|c|}{ Prueba 3 (Presión atmosférica) } \\
Orden & $\begin{array}{c}\text { Duración } \\
\text { (minutos) }\end{array}$ & $\begin{array}{c}\text { Corriente } \\
\text { (amperios) }\end{array}$ & $\begin{array}{c}\text { Voltaje } \\
\text { (voltios) }\end{array}$ & $\begin{array}{c}\text { Potencia } \\
\text { (Watts) }\end{array}$ \\
\hline Resistencia central & 4 & 2 & 65,9 & 131,8 \\
\hline Resistencia lateral & 4 & 2 & 109,8 & 219,6 \\
\hline Resistencia lateral & 4 & 2 & 80 & 160 \\
\hline
\end{tabular}

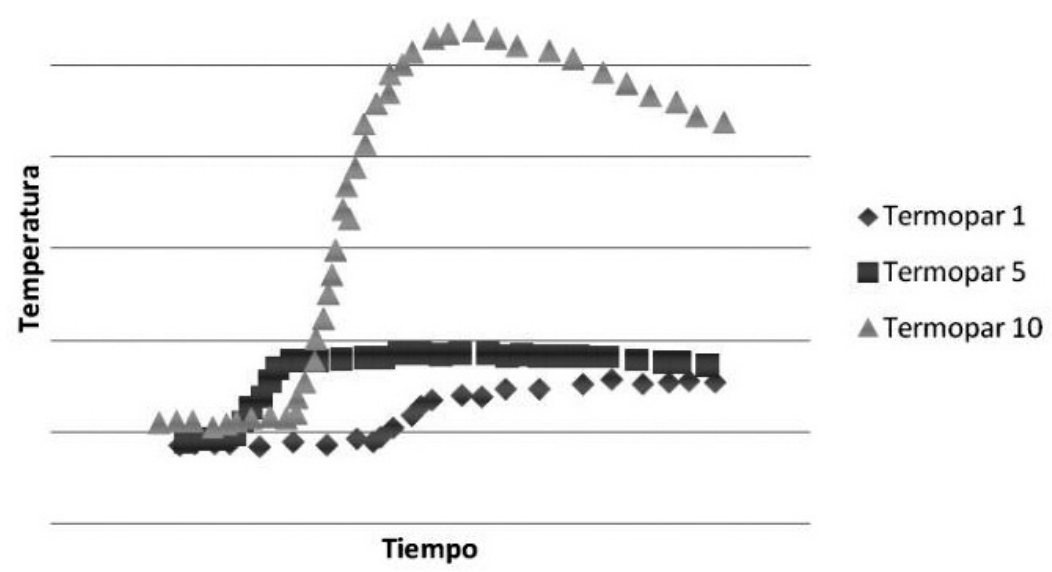

Figura 7. Gráfico de temperatura contra tiempo en la primera corrida experimental. Fuente: Ad Astra Rocket Company (comunicación personal, I de Abril de 2007).

que las resistencias dejan de entregar su potencia. La selección de los termopares 5, I, y 10 en el sistema obedece al hecho de que estos tres puntos muestran el comportamiento que se tiene tanto en la parte media como en los extremos del tubo de gases respectivamente, creando así un panorama general del registro de datos en los experimentos. Aspectos como la explicación del termopar 10 y cómo crece más en temperatura que el resto, serán parte de la labor que la compañía realice con ayuda del sistema de calentamiento. Todo lo anterior finalmente muestra que el sistema cumple sus propósitos de manera eficaz.
Las resistencias que se encuentran más cercanas a la longitud de $30 \mathrm{~cm}$ son las que generan más potencia. La resistencia central tiene más probabilidades de estar comprimida, por lo que al existir espiras en cortocircuito, existe una menor capacidad de producir calor, por lo que la potencia disipada es baja en comparación con las resistencias laterales. También se nota cómo al aplicar el amperaje en el sistema, inicialmente se mantiene, hasta que la temperatura actúa en las mismas resistencias. Como la resistencia de un material aumenta proporcionalmente con la temperatura, los valores de corriente que se establecieron al inicio descendieron conforme el tiempo 


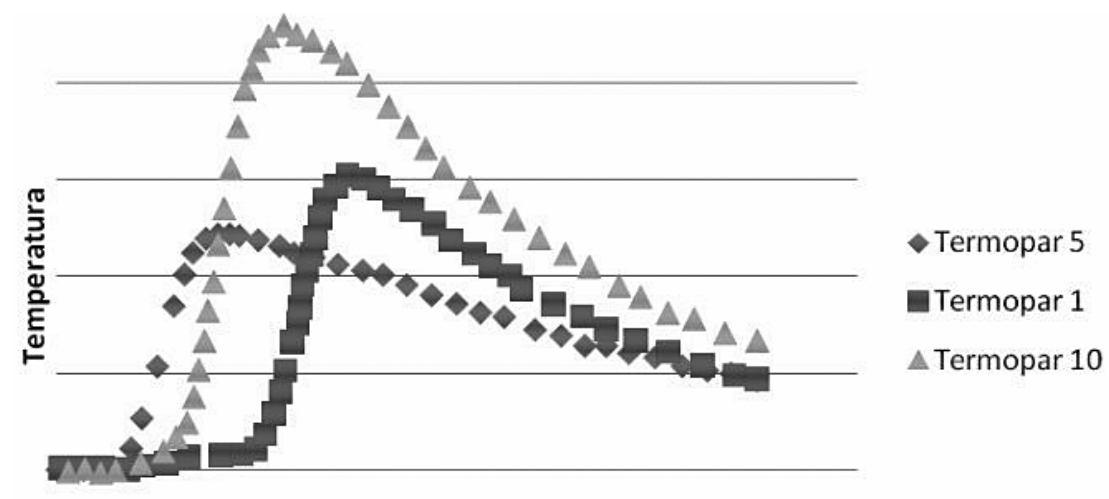

Tiempo

Figura 8. Gráfico de temperatura contra tiempo en la segunda corrida experimental. Fuente: Ad Astra Rocket Company (comunicación personal, I de Abril de 2007).

iba transcurriendo. Estos casos por analizar, así como el futuro uso que se haga de este aparato hacen ver su utilidad. El equipo profesional de la compañía será el encargado de seguir la toma de datos y la formulación de un modelado asistido por computadora.

Posteriormente, se determinará si el tubo de alúmina será un buen candidato como material del tubo de gases del motor de plasma VASIMRß, o si es necesario evaluar también otra opción con este método. El modelado por computadora básicamente será una herramienta que compare las mediciones hechas en esta etapa experimental, para analizar el comportamiento del tubo ya sea con temperaturas superiores que se deseen examinar o con condiciones de mayor o menor vacío. La

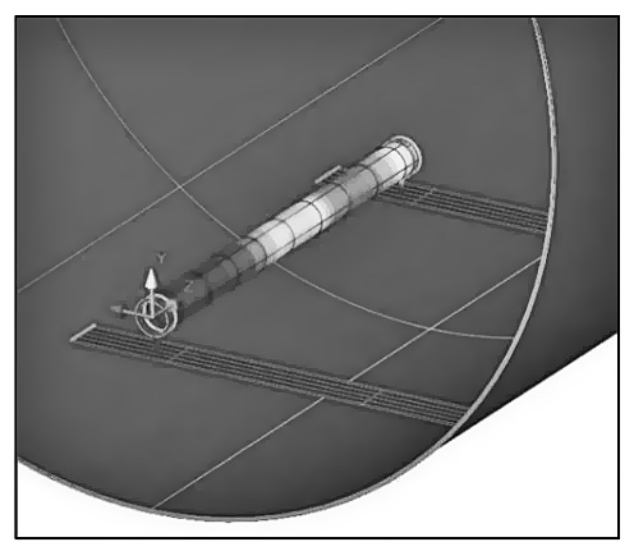

Figura 9. Modelado del tubo por computadora. Fuente: Ad Astra Rocket Company (comunicación personal, I de Abril de 2007). herramienta computacional Termal Desktop ${ }^{\circledR}$ será la encargada de esta fase, generando esquemas del tubo similares al que se ejemplifica en la Figura 9. En ella se aprecia la forma en que el tubo y la cámara de vacío que lo contiene son representados con una serie de colores que indican la temperatura a la cual se encuentran, de acuerdo con los datos que se obtuvieron de los experimentos. Este paquete computacional puede presentar gráficamente esta información así como modelar el tubo con otra serie de condiciones (sin vacío, con otro orden de calentamiento, etc.), siendo los datos obtenidos una guía valiosa para basar estas aproximaciones.

Una vez mostrada la forma de diseño e instalación del sistema de calentamiento y su efectividad a la hora de calentar el tubo, se nota como los objetivos propuestos al inicio del trabajo han sido satisfactorios. La empresa cuenta ahora con un nuevo instrumento de trabajo que será de gran ayuda en sus investigaciones, las cuales, como se mencionó al inicio, apuestan a una gran revolución en el transporte espacial para beneficio del planeta.

\section{Conclusiones}

En el trabajo realizado se cumplió a cabalidad con lo estipulado en cuanto a las restricciones del diseño y con los objetivos. Respecto al sistema de calentamiento en general, se pueden concluir los siguientes aspectos:

El plasma obedece a las leyes generales de los gases, por lo que dentro del tubo de alúmina el flujo del 
gas ionizado estará en contacto con toda la parte interna del tubo. El hecho de que en el sistema de calentamiento se escogiera una configuración de resistencias que tuviera contacto en casi toda la extensión interior del tubo valida el modelo, ya que el calentamiento no se realiza solamente en algunos puntos sino que se encuentra en contacto en casi toda la parte interna del tubo, al igual que lo estará el plasma.

La cantidad de energía para un uso nominal de 200 Watts fue superada, ya que el diseño de las resistencias fue de 975 Watts en promedio, con un sistema de control que permite tanto el uso con esa cantidad de potencia así como otras inferiores. Esto da un aumento en la temperatura experimental y un mejor acercamiento al comportamiento real del tubo, el cual ronda los $600^{\circ} \mathrm{C}$, tal y como se explicó anteriormente.

Las resistencias y demás componentes sometidos al vacío fueron seleccionados en buena forma, ya que se utilizó el sistema y no se presentaron problemas por esta condición. Los aislantes cerámicos individuales, así como las borneras cerámicas, garantizan una mayor durabilidad del cableado y una menor probabilidad de falla en el sistema.

Al tener el sistema de control la opción de dar más potencia en una zona que en otras, se contempla la posición de la antena de radiofrecuencias Hélicon, ya que ésta, a la hora de calentar el gas para ionizarlo, genera un poco más de temperatura en la zona central (donde está ubicada) que en los alrededores. Esta opción hace aún más real el diseño.

El diseño de las resistencias fue adecuado al tubo en la medida de lo posible, ya que las complicaciones surgidas a la hora de hacer una colocación por igual no permitieron que el diseño original se cumpliera del todo. Sin embargo, como se conocen las posiciones de las resistencias en realidad, se sabe cuál es la que calienta cada tramo del tubo y el modelado se puede realizar sin ningún inconveniente.

Se observó cómo al hacer la prueba con presión atmosférica, las resistencias calentaban al tubo más rápido. Se nota cómo esta herramienta de trabajo es útil para examinar el comportamiento del tubo de gases y estudiar a fondo las reacciones térmicas al vacío.

La prevista de toma de datos del sistema está contemplada en la bornera de la etapa de control, ya que de ahí se pueden obtener las lecturas que se le envían a las resistencias de voltaje y corriente, y de ellas se puede obtener la potencia.

La opción de "control remoto" en el sistema de control es de suma utilidad, ya que da mucha comodidad a la hora de ejecutar el experimento y permite un ahorro de material bastante considerable al construir un sistema de este tipo.

\section{Agradecimientos}

Al personal de la empresa Ad Astra Rocket Company Costa Rica, por su cooperación y apoyo total para la realización de este proyecto.

\section{Bibliografía}

Chang Díaz, F. (200I). An overview of the VASIMR engine: high power space propulsión with RF plasma generation and heating. American Institute of Physics 0-7354-0038-5. Recopilado el 30 de setiembre de 2006 de la base de datos EBSCO.

Díaz, C. (2005). Aceleradores de partículas. Obtenido el 30 de diciembre de 2006 desde: http://perso.wanadoo.es/chyryes/ glosario/acel_par.htm

Fallas, J.C. (2007). Diseño del sistema de calentamiento para simulación del flujo de plasma en tubo cilíndrico. Memoria para optar al Título de Licenciatura, Escuela de Ingeniería Mecánica, Universidad de Costa Rica.

Frank-Kamenetskii, D.A. (1972). Plasma, the fourth state of matter. New York: Plenum Press.

Hyndman (200I). Resistance heating wire níkel-chromium alloy $60 \%$ niquel/ $16 \%$ chromium - N6. Obtenido el 15 de marzo de 2007 desde: http://www.resistancewire.com

Lesker, K. (2006). Power Feedthroughs: CF Flange. Obtenido el 30 de diciembre de 2006 desde: http://www.lesker.com/ newweb/Feedthroughs/Electrical_Feedthroughs/Power_ Feedthrough/500V_IOA_CFFlange.cfm

MOR Electric Heating Assoc. Inc. (2007). Custom open coil element metal wire heaters. Obtenido el I 5 de marzo de 2007 desde: http://www.infraredheaters.com/metal.htm 